\title{
Meta
}

Journal des traducteurs

Translators' Journal

\section{Tommola, Jorma (Ed.) (1995) : Topics in Interpreting Research, University of Turku, Centre for Translation and Interpreting, $144 \mathrm{p}$.}

\section{Georges L. Bastin}

Volume 42, numéro 4, décembre 1997

URI : https://id.erudit.org/iderudit/001998ar

DOI : https://doi.org/10.7202/001998ar

Aller au sommaire du numéro

Éditeur(s)

Les Presses de l'Université de Montréal

ISSN

0026-0452 (imprimé)

1492-1421 (numérique)

Découvrir la revue

Citer ce compte rendu

Bastin, G. L. (1997). Compte rendu de [Tommola, Jorma (Ed.) (1995) : Topics in

Interpreting Research, University of Turku, Centre for Translation and

Interpreting, 144 p.] Meta, 42(4), 738-740. https://doi.org/10.7202/001998ar

Ce document est protégé par la loi sur le droit d'auteur. L'utilisation des services d'Érudit (y compris la reproduction) est assujettie à sa politique d'utilisation que vous pouvez consulter en ligne.

https://apropos.erudit.org/fr/usagers/politique-dutilisation/
Cet article est diffusé et préservé par Érudit.

Érudit est un consortium interuniversitaire sans but lucratif composé de l’Université de Montréal, l'Université Laval et l'Université du Québec à Montréal. Il a pour mission la promotion et la valorisation de la recherche. https://www.erudit.org/fr/ 
TOMMOLA, Jorma (Ed.) (1995) : Topics in Interpreting Research, University of Turku, Centre for Translation and Interpreting, $144 \mathrm{p}$.

L'Université de Turku, l'un des centres les plus en vue en matière d'innovation pédagogique et de recherche en traduction/interprétation, nous offre ici un recueil de communications prononcées à la conférence internationale tenue à Turku en août 1994.

L'éventail est aussi vaste que varié puisque l'interprétation (simultanée, consécutive et auprès des tribunaux) y est scrutée du point de vue pédagogique, professionnel, culturel, linguistique et cognitif, et que les auteurs viennent de Birmingham, Bologne, Grenade, Louvain, Trieste, Turku, Vienne et Warwick. Le fil conducteur en est cependant la recherche théorique et appliquée en interprétation; sa prétention, donner en exemple des sujets concrets abordés par les chercheurs.

Le premier texte, de Valeria Darò, se penche sur les aptitudes cognitives indispensables aux futurs interprètes simultanés. L'auteure part de trois modalités d'interférence de différentes composantes du processus d'interprétation, à savoir l'attention sélective, la mémoire verbale à court terme et la production verbale propre. Si l'interprète réussit à surmonter ces interférences, c'est qu'il possède certaines aptitudes ou que certaines stratégies ont été mises en œuvre durant sa formation. Comment identifier et mesurer ces aptitudes? Darò propose trois outils : l'empan mnésique, la facilité d'élocution et la lecture à haute voix. Bref, diagnostic utile mais qui demande à être traduit et évalué dans la pratique pédagogique.

L'interprétation auprès des tribunaux est l'objet de l'étude réalisée par Peter Jansen. Sur fond des normes traductionnelles de Toury et de la théorie skopos de Vermeer, Jansen décortique avec force détails la situation de l'interprète aux prises avec l'inculpé (hispanophone), le procureur et le juge durant deux procès intervenus en 1991 aux PaysBas. Le but est de confirmer la subordination des stratégies de l'interprète à la situation dans laquelle il se trouve et plus particulièrement aux circonstances dans lesquelles prend place l'interaction des divers participants. Après une description détaillée du déroulement d'un procès, l'auteur illustre judicieusement l'adaptation de l'interprète aux diverses circonstances et l'adéquation de son discours. Les exemples sont nombreux et éloquents ; la discussion est riche et convaincante. La prétendue neutralité de l'interprète judiciaire est bel et bien une illusion! 
Deux chercheurs de Bologne nous donnent ensuite les résultats d'une enquête effectuée auprès de «consommateurs» d'interprétation simultanée, à l'occasion de cinq conférences (générales et spécialisées, en allemand, français et italien). Les auteures s'attardent trop sur les questions méthodologiques posées par l'enquête; ces dernières sont certes loin d'être négligeables, mais la patience du lecteur n'est guère récompensée. Et puis, n'aurait-il pas été possible d'annexer le questionnaire employé?

Le travail suivant, de Viera Makarová, sur l'interprète dans son rôle de «médiateur interculturel» n'apprend pas grand-chose au traductologue averti, si ce n'est une certaine idiosyncrasie slovaque et les quatre dimensions de la culture de Hofstede. Ce discours généralisant surprend dans un volume consacré à des sujets bien cernés.

L'équipe de l'Université de Grenade présente une recherche de loin plus solide quoique les résultats ne soient encore que provisoires. L'étude fait partie d'un vaste projet, mené de front par des interprètes et des psychologues, sur les aptitudes cognitives et plus précisément la mémoire active mises en ouvre par les praticiens de l'interprétation simultanée. La comparaison de la capacité mnésique de professionnels, d'étudiants et de non interprètes révèle la supériorité des premiers, partant, la place qu'occupe la mémoire dans le processus d'interprétation et finalement le besoin de la développer par la pratique. La question reste cependant de savoir si ces expériences ne devraient pas porter moins sur la mémorisation de chiffres et de mots et davantage sur des unités de sens...

L'étude suivante, de Pochhacker, bien connu pour la rigueur de ses travaux, est un modèle méthodologique. En comparant la production verbale de conférenciers et d'interprètes, il tente de prouver que les interprètes commettent davantage de lapsus et d'incorrections grammaticales que les premiers. Les résultats sont maigres malgré l'ampleur du corpus (145 textes spécialisés en allemand et anglais, soit 14 heures d'enregistrement) et la rigueur de la méthode. L'auteur en explique les raisons mais souligne le potentiel de la recherche en la matière.

Christina Schäffner nous propose ensuite une classification des stratégies employées en interprétation consécutive, par des étudiants de dernière année en études allemandes, afin de combler leurs lacunes de «savoir partagé». L'intérêt de cette étude est mince si l'on considère la visée non professionnelle de la formation des sujets, le caractère artificiel des dialogues à interpréter (créés de toutes pièces pour les besoins de la cause) et la banalité des commentaires pédagogiques.

La question de savoir s'il convient à l'interprète simultané de «tout dire» ou de condenser est le sujet traité par Marianna Sunnari. L'auteure confirme que la condensation effectuée en interprétation simultanée de qualité résulte d'un «macro traitement» du message à communiquer, que l'interprète réalise au moyen d'opérations mentales visant à construire une «macro-structure» selon le modèle de Van Dijk. (Voir l'article de Mackintosh traitant de la même question dans Meta 30 (1): 37-43.) Une fois encore, l'étude est une comparaison de conférences et leur interprétation (anglais-finlandais) par des professionnels et des étudiants. Malgré la naïveté de la question de départ, une recherche plus approfondie pourrait déboucher sur une pratique pédagogique plus raisonnée. C'est d'ailleurs ce que souhaite Sunnari.

Le texte de Tommola et Lindhom soulève une interrogation majeure, celle du choix d'une «variable dépendante» pour la recherche expérimentale en interprétation. Pour ces auteurs, une variable appropriée serait l'analyse du contenu propositionnel. Ils examinent donc l'opportunité de cette variable dans la recherche des effets produits par les «variables indépendantes» (par ex., la situation, les intervenants, les tâches, le texte original). L'étude de cas présentée porte sur l'analyse propositionnelle de l'interprétation (anglaisfinlandais) de quatre textes («fabriqués») par huit interprètes chevronnés; les variantes indépendantes étant la qualité acoustique et visuelle dont bénéficient les interprètes. La 
rigueur méthodologique est exemplaire; à noter également une analyse des antécédents riche et éloquente.

L'ouvrage se termine sur une expérience menée par Tonelli et Riccardi à 1'Université de Trieste: le repérage et la correction d'erreurs lexicales, morphologiques et phonologiques par des étudiants soumis au «shadowing». L'étude révèle des stratégies tant déductives qu'inductives («top-down»/ «bottom-up») et remet de l'eau au moulin de la technique du «shadowing» comme instrument pédagogique.

GEORGES L. BASTIN

Université Centrale du Venezuela, Caracas, Venezuela 